\title{
On the Dynamics of an Impulsive Model of Hematopoiesis
}

\author{
C. Kou ${ }^{1, * \dagger}$, M. Adimy ${ }^{2}$ and A. Ducrot ${ }^{3}$ \\ ${ }^{1}$ Department of Applied Mathematics, Donghua University \\ Shanghai 201620, P. R. China \\ ${ }^{2}$ Laboratoire de Mathématiques Appliquées, UMR CNRS 5142 \& INRIA, ANUBIS \\ Université de Pau, 64000 Pau, France \\ ${ }^{3}$ Institut Mathématiques de Bordeaux, UMR CNRS 5251 \& INRIA, ANUBIS \\ Université Victor Segalen Bordeaux 2, 33076 Bordeaux, France
}

\begin{abstract}
We propose and analyze a nonlinear mathematical model of hematopoiesis, describing the dynamics of stem cell population subject to impulsive perturbations. This is a system of two age-structured partial differential equations with impulses. By integrating these equations over the age, we obtain a system of two nonlinear impulsive differential equations with several discrete delays. This system describes the evolution of the total hematopoietic stem cell populations with impulses. We first examine the asymptotic behavior of the model in the absence of impulsions. Secondly, we add the impulsive perturbations and we investigate the qualitative behavior of the model including the global asymptotic stability of the trivial solution and the existence of periodic solution in the case of periodic impulsive perturbations. Finally, numerical simulations are carried out to illustrate the behavior of the model. This study maybe helpful to understand the reactions observed in the hematopoietic system after different forms of stress as direct destruction by some drugs or irradiation.
\end{abstract}

Key words: model of hematopoiesis; impulsion; delay; asymptotic stability; Lyapunov functional; periodic solution

AMS subject classification: 34D20, 34K13, 34K45, 34K60, 92C37

\footnotetext{
* This work is supported by the NNSF of China (No. 10701 023) and the Anubis project of INRIA (France)

†Corresponding author. E-mail: kouchunhai@dhu.edu.cn
} 


\section{Introduction}

All blood cells (red cells, white cells, and platelets) are produced in the bone marrow by so-called hematopoietic stem cells. They are pluripotent stem cells, with self-renewal and differentiation abilities. Throughout divisions, hematopoietic stem cells produce an increasing number of differentiated cells (with some well known stages, such as progenitor cells, precursor cells) until the process leads to mature cells. These latter finally reach the blood stream and become blood cells.

According to the study of Burns and Tannock [8], the life of each pluripotent stem cell can be divided into two distinct phases: resting phase $\left(G_{0}\right)$ and proliferating phase $\left(G_{1}-S-G_{2}-M\right)$. The resting phase, or $G_{0}$-phase, is a quiescent stage in the cellular development. Resting cells can not divide. They can enter the proliferating phase, provided that they do not die. The proliferating phase is the active part of the cellular development. As soon as cells enter the proliferating phase, they are committed to divide, during mitosis. After division, each cell gives birth to two daughter cells which enter immediately the resting phase, and complete the cycle. Proliferating cells can also die without ending the cycle.

It is now well known that the production of blood cells is regulated by negative feedback controls, mediated by hormones. However, the exact nature of these regulatory processes is still not well known at this time. However, the work of Sachs [28] shows that we can reasonably think that it strongly depends on the entire resting cell population, that is, $\beta=\beta(N(t))$, with $N(t)$ the total population of resting cells at time $t$. For the justification of the dependence of $\beta$ on the total $G_{0}$-population $N$, see the works of Mackey and his collaborators $[6,7,9,10,15,19,20,21]$.

Due to the number of divisions and the quantity of cells involved in hematopoiesis, issues may arise at different cellular levels and sometimes result in abnormalities in blood cells production, causing the so-called dynamical hematological disorders. Most of these diseases seem to be due to a destabilization of the pluripotent hematopoietic stem cell compartment. Among a wide variety of these diseases are characterized by a periodic decrease in the circulating blood cells numbers, from normal to low values, sometimes barely detectable (Haurie et al., [15]). These blood cell diseases are known as periodic hematological diseases.

Mathematical modelling of hematopoietic stem cells dynamics has been extensively studied in the past 30 years, with attempts to determine causes leading to a number of periodic hematological disorders. In 1978, Mackey [19] proposed a mathematical model of hematopoietic stem cells dynamics inspired by the work of Burns and Tannock [8]. Formed by a system of two nonlinear delay differential equations, where the delay describes the average cell cycle duration, this model stressed the influence of some factors (such as the apoptotic rate, the introduction rate, the cell cycle duration) playing an important role in the appearance of periodic solutions. The model of Mackey [19] has been recently analyzed by Pujo-Menjouet and Mackey [25] and Pujo-Menjouet et al. [26] in order to prove the existence of long period oscillations, characterizing situations observed in patients with chronic myelogenous leukemia. Taking into account that all stem cells do not require the same time in cell cycle to divide, Adimy et al. [4] studied models similar to the one proposed by Mackey [19], but with a time delay distributed according to a density with compact support. They highlighted the role of a destabilization of the cell cycle duration in the appearance of oscillating solutions with long periods (see [22]). 
In 1998, Haurie et al. [15] published works about cyclical neutropenia, pointing out the main problems and questions arising in its study. They also gave a complete description of the disease. Bernard et al. [6] proposed the first model of leukopoiesis, based on a description of mechanisms involved in cyclical neutropenia. Their model consists of a system of two nonlinear differential equations with two time delays that describe the cell cycle duration and the time required by a hematopoietic stem cell to produce white blood cells by differentiation. We also mention the recent works of Colijn and Mackey $[9,10]$ which deal with a complete model of hematopoiesis taking into account the dynamics of the hematopoietic stem cell compartment as well as each of the three blood cell type (red blood cells, white cells and platelets) compartments.

As in many biological process, the evolution of the populations of hematopoietic cells can be characterized by the fact that at certain moments of time, due to different forms of stress as acute irradiation as well as instantaneous increase of the population of stem cells, their state change abruptly (see [18]). Consequently, it is natural to assume that these perturbations act instantaneously, that is, in the form of impulses.

Mathematical models of various biological processes and phenomena in the study of population dynamics, biology, biotechnology, ecology, etc, can be expressed by impulsive delay differential equations. They have attracted the interests of many researchers in the last two decades since they provide a natural description of the motion of several real-world processes which, on the one hand, depends on the past of the processes that often turns out to be the cause of phenomena substantially affecting the motion and, on the other hand, is subject to short-time perturbations whose duration is almost negligible.

Several various monographs $[13,16,17]$ and many papers $[12,29,31,32]$ devoted to the impulsive differential equations and impulsive delay differential equations, have been published. However, as far as we know, no works are contributed to the analysis for a model of hematopoiesis with impulsions.

In this paper, we investigate the reactions of the hematopoietic stem cell system to different forms of stress as acute irradiation as well as instantaneous increase of the population. It is commonly believed that the system must react to reach the normal state as early as possible with oscillations as small as possible.

Denote by $n(t, a)$ the population density of resting cells at time $t$ which have spent a time $a$ in their phase. Resting cells can either be lost randomly at a rate $\delta$, which takes into account the cellular differentiation, or enter into the proliferating phase at a rate $\beta$. In this proliferating phase, we suppose that cells are committed to undergo cell division at some constant times $\tau_{1}, \ldots, \tau_{m}$ after their entry. We denote by $p_{i}(t, a), i=1, \ldots, m$, the population density of proliferating cells at time $t$ which have spent a time $a$ in their phase and that divided at age $\tau_{i}$ (the point of cytokinesis). This discrete structuration of the proliferating cells corresponds to a discrete version of some distributed point of cytokinesis. The proliferating cells can be lost by apoptosis at a rate $\gamma_{i}(a)$. At the point of cytokinesis, a cell divides into two daughter cells which enter immediately the resting phase. The full model for this situation consists of a system of reaction convection evolution equations 


$$
\left\{\begin{array}{l}
\frac{\partial n}{\partial t}+\frac{\partial n}{\partial a}=-(\delta+\beta(N(t))) n, \quad t>0, a>0 \\
\frac{\partial p_{i}}{\partial t}+\frac{\partial p_{i}}{\partial a}=-\gamma_{i}(a) p_{i}, \quad t>0, \quad 0<a<\tau_{i}, \quad i=1, \ldots, m
\end{array}\right.
$$

with their associated boundary conditions

$$
\left\{\begin{array}{l}
n(t, 0)=2 \sum_{i=1}^{m} p_{i}\left(t, \tau_{i}\right), \quad t>0, \\
p_{i}(t, 0)=\int_{0}^{\infty} \alpha_{i} \beta(N(t)) n(t, a) d a=\alpha_{i} \beta(N(t)) N(t), \quad t>0, \quad i=1, \ldots, m, \\
\lim _{a \rightarrow \infty} n(t, a)=0, \quad t>0,
\end{array}\right.
$$

and initial data

$$
n(0, a)=n_{0}(a), \quad a>0, \quad p_{i}(0, a)=p_{i, 0}(a), \quad 0<a<\tau_{i}, \quad i=1, . ., m .
$$

Here $N(t)=\int_{0}^{\infty} n(t, a) d a$ denotes the total population of cells in the resting phase at time $t>0$. We assume for simplicity that the re-introduction rate $\beta(N)$ is the same for all classes $p_{i}$ weighted with some parameter $\alpha_{i} \in[0,1]$. These parameters model the repartition of the cell population coming from the resting phase into the different compartments of proliferating phase. Then, we add the condition $\sum_{i=1}^{m} \alpha_{i}=1$. We supplement our modelling by introducing some impulsions which simulate different forms of stress at some particular times $0<t_{1}<t_{2}<\ldots<t_{k}<\ldots$. These conditions can be expressed as follow

$$
N\left(t_{k}^{+}\right)=\left(1+b_{k}\right) N\left(t_{k}\right), \quad k=1,2, \ldots,
$$

where the sequence $\left\{b_{k}\right\}_{k \geq 1}$ is such that $b_{k}>-1$, for $k \geq 1$. The condition (1.4) corresponds to instantaneous perturbations of the system by adding $\left(b_{k}>0\right)$ or losing $\left(-1<b_{k}<0\right)$ hematopoietic stem cells.

In order to tackle (1.1)-(1.4) by using delay differential equations, we assume, for simplicity, that the functions $\gamma_{i}(a) \equiv \gamma_{i}$ are constants. Then by employing the method of characteristics [30] and the boundary conditions (1.2), we obtain

$$
p_{i}\left(t, \tau_{i}\right)=\alpha_{i} e^{-\gamma_{i} \tau_{i}} \beta\left(N\left(t-\tau_{i}\right)\right) N\left(t-\tau_{i}\right), \quad t>\tau_{i}, \quad i=1, . ., m .
$$

By integrating (1.1) over the age and using (1.5), the model (1.1) can be transformed into a system of nonlinear first-order delay differential equations [19, 20, 21]

$$
\begin{gathered}
\frac{d P_{i}(t)}{d t}=-\gamma_{i} P_{i}(t)+\beta(N(t)) N(t)-\alpha_{i} e^{-\gamma_{i} \tau_{i}} \beta\left(N\left(t-\tau_{i}\right)\right) N\left(t-\tau_{i}\right), \\
\frac{d N(t)}{d t}=-(\delta+\beta(N(t))) N(t)+2 \sum_{i=1}^{m} \alpha_{i} e^{-\gamma_{i} \tau_{i}} \beta\left(N\left(t-\tau_{i}\right)\right) N\left(t-\tau_{i}\right),
\end{gathered}
$$


with $P_{i}(t)=\int_{0}^{\tau} p_{i}(t, a) d a, t>0$. This system is valid for $t>\tau:=\max _{i=1, . ., m}\left(\tau_{i}\right)$. Up to a time translation, we consider this system posed for $t>0$ and with associated initial conditions defined on $[-\tau, 0]$.

The function $\beta$ is supposed to be continuous and positive. Furthermore, from a reasonable biological point of view, we assume that $\beta$ is decreasing with $\lim _{x \rightarrow+\infty} \beta(x)=0$. This describes the fact that the rate of reentry into the proliferating compartment is a decreasing function of the $G_{0}$-phase population ([19], [28]). Usually, it is believed that the function $\beta$ is a monotone decreasing Hill function (see Mackey [19]), given by

$$
\beta(N)=\beta_{0} \frac{\theta^{n}}{\theta^{n}+N^{n}},
$$

where $\beta_{0}$ is the maximal rate of cell movement from $G_{0}$ into the proliferating phase, $\theta$ is the $G_{0}$ stem cells population at which the rate of cell movement from $G_{0}$ into proliferation is one-half of its maximal value $\left(\beta_{0}\right)$, and $n>0$ characterizes the sensitivity of the mitotic reentry rate $\beta$ with changes in the size of $G_{0}$-phase population. The Hill function $\beta(N)$ has often been used in cell cycle models to describe saturation [11].

It should be pointed out that the dynamics of the resting phase cells described by (1.7) are independent of the dynamics of the proliferative phase cells, but the converse is not true. This is due to the assumed dependence of $\beta$ on $N$ as well as the boundary conditions of the original equations of the problem $[19,20]$. Because of this independence, we are concerned only with equation (1.7).

More precisely, we investigate the following model of hematopoiesis with impulsions

$$
\left\{\begin{array}{l}
x^{\prime}(t)=-(\delta+\beta(x(t))) x(t)+2 \sum_{i=1}^{m} \alpha_{i} e^{-\gamma_{i} \tau_{i}} \beta\left(x\left(t-\tau_{i}\right)\right) x\left(t-\tau_{i}\right), \quad t \neq t_{k}, \\
x\left(t_{k}^{+}\right)=\left(1+b_{k}\right) x\left(t_{k}\right), \quad k=1,2, \ldots \\
x(t)=\varphi(t), \quad t \in[-\tau, 0]
\end{array}\right.
$$

where $x\left(t_{k}^{+}\right)=\lim _{t \rightarrow t_{k}^{+}} x(t), k \geq 1$.

The paper is organized as follows. In Section 2, we consider the delay system (1.9) without impulsions, and present some results about the stationary solutions together with their stabilities. Section 3 investigates system (1.9) in presence of impulsions. We first reduce the impulsive equation to a non-impulsive one and obtain well posedness properties for the associated Cauchy problem. Then the global asymptotic stability of the trivial solution is studied. The case of $\omega$-periodic impulsions is considered. Some conditions for the existence of a unique $\omega$-periodic solution of system (1.9) are given. Finally, numerical simulations illustrate the behavior of the model. 


\section{Delay differential equations without impulsions}

In this section, we consider equation (1.9) without impulsions. This particular case corresponds to $b_{k}=0$ for all $k \geq 1$. Therefore, we have the following equation

$$
\left\{\begin{array}{l}
x^{\prime}(t)=-(\delta+\beta(x(t))) x(t)+2 \sum_{i=1}^{m} \alpha_{i} e^{-\gamma_{i} \tau_{i}} \beta\left(x\left(t-\tau_{i}\right)\right) x\left(t-\tau_{i}\right), \quad t>0, \\
x(t)=\varphi(t), \quad t \in[-\tau, 0]
\end{array}\right.
$$

where $\varphi \in C\left([-\tau, 0], \mathbb{R}^{+}\right)$is an initial condition and $\tau=\max _{i=1, \ldots, m}\left\{\tau_{i}\right\}$. Equation (2.1) has been studied by Pujo-Menjouet et al. in [25, 26] with one discrete delay. Here we extend some of these results to multiple discrete delays. We first have the following well posedness result.

Theorem 1. For each nonnegative initial condition $\varphi \in C\left([-\tau, 0], \mathbb{R}^{+}\right)$, equation (2.1) has a unique nonnegative and bounded solution.

The proof of this result is classical and follows the same arguments as the ones given below in the proof of Theorem 7.

We now consider the equilibria of equation (2.1). An equilibrium $x^{*} \geq 0$ is a time-independent solution. This means that $x^{*}$ is a solution of the problem

$$
-\left(\delta+\beta\left(x^{*}\right)\right) x^{*}+2 \sum_{i=1}^{m} \alpha_{i} e^{-\gamma_{i} \tau_{i}} \beta\left(x^{*}\right) x^{*}=0 .
$$

We first concentrate ourselves on the existence of equilibria and their asymptotic stability. Notice that $x^{*}=0$ is always an equilibrium of equation (2.1). It describes the extinction of the cell populations. Searching for positive equilibrium of (2.1), that is $x^{* *}>0$, we must solve

$$
\delta-\left(2 \sum_{i=1}^{m} \alpha_{i} e^{-\gamma_{i} \tau_{i}}-1\right) \beta\left(x^{* *}\right)=0
$$

We get the theorem.

Theorem 2. Assume that $\delta>0$. Then the following results hold.

(i) If

$$
\delta>\beta(0)\left(2 \sum_{i=1}^{m} \alpha_{i} e^{-\gamma_{i} \tau_{i}}-1\right)
$$

then $x^{*}=0$ is the unique equilibrium solution of (2.1).

Moreover, this equilibrium is globally asymptotically stable. 
(ii) If we suppose that

$$
\delta<\beta(0)\left(2 \sum_{i=1}^{m} \alpha_{i} e^{-\gamma_{i} \tau_{i}}-1\right)
$$

then (2.1) has two equilibria $x^{*}=0$ and $x^{* *}>0$. In this case, the trivial equilibrium $x^{*}=0$ is unstable. If in addition we have

$$
\bar{\beta}=\frac{d}{d x}(x \beta(x))_{\mid x=x^{* *}}>0,
$$

then the positive equilibrium $x^{* *}$ is locally asymptotically stable (LAS).

Remark 3. When $\delta$ crosses the value $\beta(0)\left(2 \sum_{i=1}^{m} \alpha_{i} e^{-\gamma_{i} \tau_{i}}-1\right)$, the dynamical system undergoes a transcritical bifurcation with a change of stability of the trivial state $x^{*}=0$.

Proof. We first investigate the existence of positive equilibrium. It satisfies the equation

$$
\delta-\left(2 \sum_{i=1}^{m} \alpha_{i} e^{-\gamma_{i} \tau_{i}}-1\right) \beta\left(x^{* *}\right)=0 .
$$

Since $\beta$ is a decreasing map with image $(0, \beta(0)]$, we obtain that this last equation has a solution $x^{* *}>0$ if and only if (2.3) is satisfied.

We now consider the stability of the equilibria.

First suppose that (2.2) is satisfied. Then we consider the Lyapunov functional

$$
V(\varphi)=\varphi(0)+2 \sum_{i=1}^{m} \alpha_{i} e^{-\gamma_{i} \tau_{i}} \int_{-\tau_{i}}^{0} \varphi(\theta) \beta(\varphi(\theta)) d \theta, \quad \varphi \in C\left([-\tau, 0], \mathbb{R}^{+}\right) .
$$

If $t \rightarrow x(t)$ is a solution of (2.1), we have

$$
V\left(x_{t}\right)=x(t)+2 \sum_{i=1}^{m} \alpha_{i} e^{-\gamma_{i} \tau_{i}} \int_{t-\tau_{i}}^{t} x(\theta) \beta(x(\theta)) d \theta, \quad t>0 .
$$

Here $x_{t}, t \geq 0$, denotes the history function defined by $x_{t}(\theta)=x(t+\theta)$ for $\theta \in[-\tau, 0]$. We obtain, for $t>0$,

$$
\begin{aligned}
\frac{d}{d t} V\left(x_{t}\right)= & -[\delta+\beta(x(t))] x(t)+2 \sum_{i=1}^{m} \alpha_{i} e^{-\gamma_{i} \tau_{i}} \beta\left(x\left(t-\tau_{i}\right)\right) x\left(t-\tau_{i}\right)+ \\
& 2 \sum_{i=1}^{m} \alpha_{i} e^{-\gamma_{i} \tau_{i}}\left[\left(x(t) \beta(x(t))-\beta\left(x\left(t-\tau_{i}\right)\right) x\left(t-\tau_{i}\right)\right)\right] \\
= & -\left[\delta-\left(2 \sum_{i=1}^{m} \alpha_{i} e^{-\gamma_{i} \tau_{i}}-1\right) \beta(x(t))\right] x(t) .
\end{aligned}
$$


We now set the map

$$
u(x)=\left[\delta-\left(2 \sum_{i=1}^{m} e^{-\gamma_{i} \tau_{i}}-1\right) \beta(x)\right] x .
$$

Since $\beta(x) \leq \beta(0)$ for each $x \geq 0$ and due to condition (2.2), we get $u(x) \geq 0$ for each $x \geq 0$ and

$$
\frac{d}{d t} V(\varphi)=-u(\varphi(0)), \quad \varphi \in C\left([-\tau, 0], \mathbb{R}^{+}\right)
$$

Since $\beta$ is decreasing, the function $u$ satisfies the propriety $u(x)=0$ if and only if $x=0$. The point (i) is now complete.

We now prove (ii). We first show the instability of $x^{*}=0$. For that purpose we look at the characteristic equation corresponding the linearized equation about the zero equilibrium. This characteristic equation reads

$$
\Delta(\lambda)=\lambda+[\delta+\beta(0)]-2 \sum_{i=1}^{m} \alpha_{i} e^{-\left(\gamma_{i}+\lambda\right) \tau_{i}} \beta(0), \quad \lambda \in \mathbb{C} .
$$

Recall that the roots of the equation $\Delta(\lambda)=0$ provide the eigenvalues of the linearized equation about $x^{*}=0$ and thus control the stability of this equilibrium.

The map $g_{1}: \lambda \in \mathbb{R} \rightarrow \lambda+[\delta+\beta(0)]$ is increasing while the map $g_{2}: \lambda \in \mathbb{R} \rightarrow 2 \sum_{i=1}^{m} \alpha_{i} e^{-\left(\gamma_{i}+\lambda\right) \tau_{i}} \beta(0)$ is decreasing. Thus under condition (2.3) we have

$$
g_{1}(0)<g_{2}(0)
$$

and we can conclude that the equation $\Delta(\lambda)=0$ has a solution in the interval $(0, \infty)$. This complete the proof of the instability result.

It remains to prove that under conditions (2.3) and (2.4) the point $x^{* *}$ is LAS. First, recall that $x^{* *}$ satisfies the equation

$$
\delta-\left(2 \sum_{i=1}^{m} \alpha_{i} e^{-\gamma_{i} \tau_{i}}-1\right) \beta\left(x^{* *}\right)=0 .
$$

Then the local stability of this point is given by the roots of the corresponding characteristic equation. It easily reads as

$$
\bar{\Delta}(\lambda)=\lambda+\delta+\bar{\beta}-2 \bar{\beta} \sum_{i=1}^{m} \alpha_{i} e^{-\left(\gamma_{i}+\lambda\right) \tau_{i}} .
$$

Let us first notice that under the condition $\bar{\beta}>0$, the map $\lambda \in \mathbb{R} \rightarrow \bar{\Delta}(\lambda)$ is increasing. Indeed we have

$$
\frac{d \bar{\Delta}}{d \lambda}(\lambda)=1+2 \bar{\beta} \sum_{i=1}^{m} \tau_{i} \alpha_{i} e^{-\left(\gamma_{i}+\lambda\right) \tau_{i}}>0, \quad \lambda \in \mathbb{R} .
$$

Moreover, we have

$$
\lim _{\lambda \rightarrow-\infty} \bar{\Delta}(\lambda)=-\infty, \lim _{\lambda \rightarrow+\infty} \bar{\Delta}(\lambda)=+\infty
$$


Now, if we compute $\bar{\Delta}(0)$ we get

$$
\begin{aligned}
\bar{\Delta}(0) & =\delta+\bar{\beta}-2 \bar{\beta} \sum_{i=1}^{m} \alpha_{i} e^{-\gamma_{i} \tau_{i}} \\
& =\left(1-2 \sum_{i=1}^{m} \alpha_{i} e^{-\gamma_{i} \tau_{i}}\right)\left(x^{* *} \beta^{\prime}\left(x^{* *}\right)\right)>0 .
\end{aligned}
$$

Thus, there exists $\lambda_{0} \in(-\infty, 0)$ such that $\bar{\Delta}\left(\lambda_{0}\right)=0$. Let us now consider a general solution $\lambda=\mu+i \nu \in \mathbb{C}$ of the characteristic equation $\bar{\Delta}(\lambda)=0$. We will prove that $\mu \leq \lambda_{0}<0$. Since $\lambda_{0}$ and $\lambda$ are solutions of the characteristic equation, we have

$$
\left\{\begin{array}{l}
\lambda_{0}+\delta+\bar{\beta}-2 \bar{\beta} \sum_{i=1}^{m} \alpha_{i} e^{-\left(\gamma_{i}+\lambda_{0}\right) \tau_{i}}=0 \\
\lambda+\delta+\bar{\beta}-2 \bar{\beta} \sum_{i=1}^{m} \alpha_{i} e^{-\left(\gamma_{i}+\lambda\right) \tau_{i}}=0 .
\end{array}\right.
$$

Then by taking the real part and by introducing $X=\lambda_{0}-\mu$, we get

$$
X=2 \bar{\beta} \sum_{i=1}^{m} \alpha_{i} e^{-\left(\gamma_{i}+\mu\right) \tau_{i}}\left(e^{-X \tau_{i}}-\cos \left(\nu \tau_{i}\right)\right) .
$$

We argue by contradiction by assuming that $X<0$. Then, for any $i=1, . ., m$, we have $e^{-X \tau_{i}}-$ $\cos \left(\nu \tau_{i}\right)>0$ which is in contradiction with $X<0$. Thus $X=\lambda_{0}-\mu \geq 0$ and we conclude that the solutions of the characteristic equation are located in the strip $\left\{\lambda \in \mathbb{C}, \Re \lambda \leq \lambda_{0}<0\right\}$. This completes the proof of Theorem 2.

Remark 4. The proof of the last theorem is inspired from the once of the result given by [4] in the case of equation with distributed delay.

\section{The impulsive delay differential equation}

This section is devoted to the study of some properties of the impulsive equation (1.9). We first derive some general properties and then we study the case of periodic forcing impulsions.

Throughout this section, we assume the following conditions.

(H1) $0<t_{1}<t_{2}<\cdots$ are fixed impulsive points such that $\lim _{k \rightarrow+\infty} t_{k}=+\infty$;

(H2) $\left\{b_{k}\right\}_{k \geq 1}$ is a real sequence such that $b_{k}>-1$, for $k=1,2, \ldots$

In the sequel, we use the map $t \rightarrow \prod_{0<t_{k}<t}\left(1+b_{k}\right)$. We always assume that this product is equal to unity if the number of factors is zero. Note that due to the assumption (H2), this map is positive. Moreover, we assume that

(H3) The map $t \rightarrow \prod_{0<t_{k}<t}\left(1+b_{k}\right)$ is bounded. 


\subsection{Reduction to a non-impulsive equation}

In this section, we re-write equation (1.9) in a non-impulsive form (in a non-autonomous delay differential form). Due to the biological motivation, we consider the system (1.9) accompanied with the initial condition

$$
x(t)=\phi(t), t \in[-\tau, 0], \quad \phi \in C([-\tau, 0],[0,+\infty)),
$$

with $\tau=\max _{1 \leq i \leq m}\left\{\tau_{i}\right\}$.

We now recall some definitions of the solutions of problem (1.9) together with an initial condition (3.1).

Definition 5. [29] For any $\phi \in C([-\tau, 0],[0,+\infty))$, a function $x$ defined on $[-\tau,+\infty)$ with values in $\mathbb{R}$ is said to be a solution of (1.9) on $[-\tau, \infty)$ if we have the following

(i) $x(t)=\phi(t), t \in[-\tau, 0]$.

(ii) $x(t)$ is absolutely continuous on each interval $\left(0, t_{1}\right]$ and $\left(t_{k}, t_{k+1}\right], k=1,2, \cdots$.

(iii) For any $k=1,2, \cdots, x\left(t_{k}^{+}\right)$and $x\left(t_{k}^{-}\right)$exist and $x\left(t_{k}^{-}\right)=x\left(t_{k}\right)$.

(iv) $x(t)$ satisfies the differential equation in (1.9) for almost everywhere $t$ in $[0,+\infty) \backslash \cup_{k \geq 1}\left\{t_{k}\right\}$ and the impulsive conditions for every $t=t_{k}, k=1,2, \cdots$.

The system (1.9) admits a unique solution for each continuous initial condition (3.1) (the proof is similar to that in Hale and Verduyn Lunel [14]).

Under Hypothesis (H1)-(H3) and the expression of the function $\beta$, we consider the nonimpulsive delay differential equation

$$
y^{\prime}(t)=-\left(\delta+\beta_{0} \frac{a^{n}(t)}{a^{n}(t)+y^{n}(t)}\right) y(t)+2 \beta_{0} \sum_{i=1}^{m} \alpha_{i} e^{-\gamma_{i} \tau_{i}} \frac{a^{n}\left(t-\tau_{i}\right) d_{i}(t)}{a^{n}\left(t-\tau_{i}\right)+y^{n}\left(t-\tau_{i}\right)} y\left(t-\tau_{i}\right),
$$

where

$$
a(t)=\theta \prod_{0<t_{k}<t}\left(1+b_{k}\right)^{-1}, d_{i}(t)=\prod_{t-\tau_{i} \leq t_{k}<t}\left(1+b_{k}\right)^{-1}, \quad i=1, \ldots, m .
$$

On can remark that

$$
a(t)=\theta \text { for } t \in[-\tau, 0] \text { and } d_{i}(t)=\frac{a(t)}{a\left(t-\tau_{i}\right)} \text { for } t \geq 0 .
$$

The equation (3.2) is supplemented with an initial condition

$$
y(t)=\phi(t), t \in[-\tau, 0], \phi \in C([-\tau, 0],[0,+\infty)) .
$$

By a solution $y(t)$ on $[-\tau,+\infty)$ of (3.2)-(3.4), we mean an absolutely continuous function $y(t)$ defined on $[-\tau,+\infty)$ satisfying (3.2)-(3.4).

The following lemma is useful in this paper. 
Lemma 6. Assume that (H1)-(H3) hold.

(i) If $x(t)$ is a solution of (1.9), then $y(t)=\prod_{0<t_{k}<t}\left(1+b_{k}\right)^{-1} x(t)$ is a solution of (3.2).

(ii) If $y(t)$ is a solution of (3.2), then $x(t)=\prod_{0<t_{k}<t}\left(1+b_{k}\right) y(t)$ is a solution of (1.9).

Proof. (i) Let $x(t)$ be a solution of (1.9). Set $y(t)=\prod_{0<t_{k}<t}\left(1+b_{k}\right)^{-1} x(t)$ for $t \in[-\tau,+\infty)$. Then, $y(t)=x(t)$ for $t \in[-\tau, 0]$. Since $x(t)$ is absolutely continuous on $\left(0, t_{1}\right]$ and on each interval $\left(t_{k}, t_{k+1}\right], k=1,2, \cdots$, and, in view of the impulsive condition, it follows that for any $k=1,2, \cdots$,

$$
y\left(t_{k}^{+}\right)=\lim _{t \rightarrow t_{k}^{+}} \prod_{0<t_{j}<t}\left(1+b_{j}\right)^{-1} x(t)=\prod_{j=1}^{k}\left(1+b_{j}\right)^{-1} x\left(t_{k}^{+}\right)=\prod_{j=1}^{k-1}\left(1+b_{j}\right)^{-1} x\left(t_{k}\right)=y\left(t_{k}\right),
$$

and

$$
y\left(t_{k}^{-}\right)=\prod_{j=1}^{k-1}\left(1+b_{j}\right)^{-1} x\left(t_{k}^{-}\right)=\prod_{j=1}^{k-1}\left(1+b_{j}\right)^{-1} x\left(t_{k}\right)=y\left(t_{k}\right) .
$$

This implies that $y(t)$ is continuous on $[-\tau,+\infty)$. Furthermore, from (1.9), for any $0<t \neq t_{k}$, $k=1,2, \cdots$, we have

$$
\begin{aligned}
\prod_{0<t_{k}<t}\left(1+b_{k}\right)^{-1} x^{\prime}(t)= & -\delta \prod_{0<t_{k}<t}\left(1+b_{k}\right)^{-1} x(t)-\beta_{0} \frac{\theta^{n}}{\theta^{n}+x^{n}(t)} \prod_{0<t_{k}<t}\left(1+b_{k}\right)^{-1} x(t) \\
& +2 \beta_{0} \sum_{i=1}^{m} \frac{\alpha_{i} e^{-\gamma_{i} \tau_{i}} \theta^{n}}{\theta^{n}+x^{n}\left(t-\tau_{i}\right)} \prod_{0<t_{k}<t}\left(1+b_{k}\right)^{-1} x\left(t-\tau_{i}\right) .
\end{aligned}
$$

Then,

$$
y^{\prime}(t)=-\left(\delta+\beta_{0} \frac{a^{n}(t)}{a^{n}(t)+y^{n}(t)}\right) y(t)+2 \beta_{0} \sum_{i=1}^{m} \alpha_{i} e^{-\gamma_{i} \tau_{i}} \frac{a^{n}\left(t-\tau_{i}\right) d_{i}(t)}{a^{n}\left(t-\tau_{i}\right)+y^{n}\left(t-\tau_{i}\right)} y\left(t-\tau_{i}\right) .
$$

This implies that $y(t)$ satisfies (3.2). Thus, $y(t)=\prod_{0<t_{k}<t}\left(1+b_{k}\right)^{-1} x(t)$ is a solution of (3.2). Remark that $y^{\prime}\left(t_{k}^{-}\right)$and $y^{\prime}\left(t_{k}^{+}\right)$exist but in general $y^{\prime}\left(t_{k}^{-}\right) \neq y^{\prime}\left(t_{k}^{+}\right), k=1, \ldots, m$.

Next, we prove (ii). Let $y(t)$ be a solution of (3.2). Set $x(t)=y(t)$ for $t \in[-\tau, 0]$ and $x(t)=\prod_{0<t_{k}<t}\left(1+b_{k}\right) y(t)$ for $t \in(0,+\infty)$. It is easy to see that $x(t)$ is absolutely continuous on $\left(0, t_{1}\right]$ and $\left(t_{k}, t_{k+1}\right], k=1,2, \cdots$, and from (3.2) for any $t \neq t_{k}, k=1,2, \cdots$, we have

$$
\begin{aligned}
\prod_{0<t_{k}<t}\left(1+b_{k}\right) y^{\prime}(t)= & -\delta \prod_{0<t_{k}<t}\left(1+b_{k}\right) y(t)-\beta_{0} \frac{a^{n}(t)}{a^{n}(t)+y^{n}(t)} \prod_{0<t_{k}<t}\left(1+b_{k}\right) y(t) \\
& +2 \beta_{0} \sum_{i=1}^{m} \alpha_{i} e^{-\gamma_{i} \tau_{i}} \frac{a^{n}\left(t-\tau_{i}\right) d_{i}(t)}{a^{n}\left(t-\tau_{i}\right)+y^{n}\left(t-\tau_{i}\right)} \prod_{0<t_{k}<t}\left(1+b_{k}\right) y\left(t-\tau_{i}\right) .
\end{aligned}
$$


That is,

$$
x^{\prime}(t)=-(\delta+\beta(x(t))) x(t)+2 \sum_{i=1}^{m} \alpha_{i} e^{-\gamma_{i} \tau_{i}} \beta\left(x\left(t-\tau_{i}\right)\right) x\left(t-\tau_{i}\right) .
$$

This implies that $x(t)$ satisfies the differential equation in (1.9).

On the other hand, for every $t_{k}, k=1,2, \cdots$,

$$
x\left(t_{k}^{+}\right)=\lim _{t \rightarrow t_{k}^{+}} \prod_{0<t_{j}<t}\left(1+b_{j}\right) y(t)=\prod_{j=1}^{k}\left(1+b_{j}\right) y\left(t_{k}\right)=\left(1+b_{k}\right) \prod_{j=1}^{k-1}\left(1+b_{j}\right) y\left(t_{k}\right),
$$

and

$$
x\left(t_{k}\right)=\prod_{0<t_{j}<t_{k}}\left(1+b_{j}\right) y\left(t_{k}\right)=\prod_{j=1}^{k-1}\left(1+b_{j}\right) y\left(t_{k}\right) .
$$

Thus, for every $k=1,2, \cdots$,

$$
x\left(t_{k}^{+}\right)=\left(1+b_{k}\right) x\left(t_{k}\right) .
$$

This implies that $x(t)$ satisfies the impulsive conditions. Hence, $x(t)$ is a solution of (1.9).

The proof of the lemma is complete.

We introduce the following notations. For a function $f:[0,+\infty) \rightarrow \mathbb{R}$, we define

$$
\bar{F}=\sup _{t \geq 0}\{f(t)\}, \quad \underline{F}=\inf _{t \geq 0}\{f(t)\} .
$$

For an $\omega$-periodic function $f$, we have $\bar{F}=\max _{0 \leq t \leq \omega}\{f(t)\}, \underline{F}=\min _{0 \leq t \leq \omega}\{f(t)\}$.

Theorem 7. Let the assumptions $(\boldsymbol{H 1})-(\mathrm{H3})$ be satisfied. The solutions of (1.9) associated with nonnegative initial conditions (3.1) are nonnegative and bounded.

Proof. We first prove that the solutions of (3.2) are nonnegative. Indeed, assume that there exists a $T>0$ and $0<\varepsilon<\min _{i=1, \ldots, m}\left\{\tau_{i}\right\}$ such that $y(t) \geq 0$ for $t<T, y(T)=0$ and $y(t)<0, y^{\prime}(t)<0$ for $T<t<T+\varepsilon$. Then from (3.2) we have, for $T<t<T+\varepsilon$,

$0>y^{\prime}(t)=-\left(\delta+\beta_{0} \frac{a^{n}(t)}{a^{n}(t)+y^{n}(t)}\right) y(t)+2 \beta_{0} \sum_{i=1}^{m} \alpha_{i} e^{-\gamma_{i} \tau_{i}} \frac{a^{n}\left(t-\tau_{i}\right) d_{i}(t)}{a^{n}\left(t-\tau_{i}\right)+y^{n}\left(t-\tau_{i}\right)} y\left(t-\tau_{i}\right)>0$,

Consequently, $y(t)$ is a nonnegative function.

Now, let us prove that the solutions of (3.2) are bounded. Define the function $g:[0,+\infty) \rightarrow$ $\mathbb{R}^{+*}$ by

$$
g(y)=2 \beta_{0} \frac{\bar{A}^{n} K}{\underline{A}^{n}+y^{n}}
$$

where

$$
K=\max _{i=1, \ldots, m}\left\{\alpha_{i} e^{-\gamma_{i} \tau_{i}} \overline{D_{i}}\right\}
$$


Recall that the notations $\bar{A}, \underline{A}$ and $\overline{D_{i}}$ mean

$$
\bar{A}=\sup _{t \geq 0}\{a(t)\}, \quad \underline{A}=\inf _{t \geq 0}\{a(t)\} \text { and } \overline{D_{i}}=\sup _{t \geq 0}\left\{d_{i}(t)\right\}
$$

Let us first notice that the function $g$ is decreasing and tend to 0 when $y$ tends to infinity. Therefore, we can find a real number $\chi_{0}>0$ such that $g(y) \leq \frac{\delta}{2 m}$ for any $y>\chi_{0}$. Thus, we have

$$
g(y) y \leq \begin{cases}g(0) \chi_{0} & \text { if } 0 \leq y \leq \chi_{0} \\ \frac{\delta}{2 m} y & \text { if } y>\chi_{0}\end{cases}
$$

Define the quantity

$$
\chi_{1}:=\frac{2 m \chi_{0}}{\delta} g(0)
$$

Let $x>\chi_{1}$ and $y \in[0, x]$ be given. Then we have

$$
g(y) y \leq \begin{cases}g(0) \chi_{0}=\frac{\delta}{2 m} \chi_{1}<\frac{\delta}{2 m} x & \text { if } 0 \leq y \leq \chi_{0} \\ \frac{\delta}{2 m} x & \text { if } y>\chi_{0}\end{cases}
$$

Assume now that $y(t)$ is unbounded. Then, one can find a $t_{0}>0$ such that

$$
0 \leq y(t) \leq y\left(t_{0}\right) \text { for } t \in\left[t_{0}-\tau, t_{0}\right] \text { and } y\left(t_{0}\right)>\chi_{1} \text {. }
$$

Thus, from (3.2) one has

$$
\begin{aligned}
y^{\prime}\left(t_{0}\right) & =-\delta y\left(t_{0}\right)-\beta_{0} \frac{a^{n}\left(t_{0}\right)}{a^{n}\left(t_{0}\right)+y^{n}\left(t_{0}\right)} y\left(t_{0}\right)+2 \beta_{0} \sum_{i=1}^{m} \alpha_{i} e^{-\gamma_{i} \tau_{i}} \frac{a^{n}\left(t_{0}-\tau_{i}\right) d_{i}\left(t_{0}\right)}{a^{n}\left(t_{0}-\tau_{i}\right)+y^{n}\left(t_{0}-\tau_{i}\right)} y\left(t_{0}-\tau_{i}\right), \\
& \leq-\delta y\left(t_{0}\right)-\beta_{0} \frac{a^{n}\left(t_{0}\right)}{a^{n}\left(t_{0}\right)+y^{n}\left(t_{0}\right)} y\left(t_{0}\right)+\sum_{i=1}^{m} g\left(y\left(t_{0}-\tau_{i}\right)\right) y\left(t_{0}-\tau_{i}\right) .
\end{aligned}
$$

Using (3.5), we get

$$
y^{\prime}\left(t_{0}\right) \leq-\delta y\left(t_{0}\right)-\beta_{0} \frac{a^{n}\left(t_{0}\right)}{a^{n}\left(t_{0}\right)+y^{n}\left(t_{0}\right)} y\left(t_{0}\right)+\frac{\delta}{2} y\left(t_{0}\right)<0 .
$$

This leads to a contradiction. We deduce that $t \rightarrow y(t)$ is bounded and the proof of the theorem is complete by using Lemma 6 .

\subsection{Global asymptotic stability of the trivial solution}

In this part, we give a sufficient condition for the global asymptotic stability of the solution $x=0$ of system (1.9). Let $V: \mathbb{R}^{+} \times C\left([-\tau, 0], \mathbb{R}^{+}\right) \rightarrow \mathbb{R}$ be the function defined by

$$
V(t, \varphi)=\varphi(0)+2 \beta(0) \sum_{i=1}^{m} \alpha_{i} e^{-\gamma_{i} \tau_{i}} \int_{-\tau_{i}}^{0} \frac{a^{n}(t+\theta) d_{i}\left(t+\theta+\tau_{i}\right)}{a^{n}(t+\theta)+\varphi^{n}(\theta)} \varphi(\theta) d \theta
$$


Then using the same technique as in the proof of Theorem 2, we obtain that the derivative of $V(t, \varphi)$ along the solutions of equation (3.2) satisfies

$$
\frac{d}{d t} V(t, \varphi) \leqslant-\left[\delta-\beta_{0}\left(2 \sum_{i=1}^{m} \alpha_{i} e^{-\gamma_{i} \tau_{i}} \overline{D_{i}}-1\right) \frac{a^{n}(t)}{a^{n}(t)+\varphi^{n}(0)}\right] \varphi(0) .
$$

By the assumption (3.6), we establish that $V$ is a Lyapunov functional. Then we obtain the following result.

Theorem 8. Assume that

$$
\delta>\beta_{0} \frac{\bar{A}^{n}}{\underline{A}^{n}}\left(2 \sum_{i=1}^{m} \alpha_{i} e^{-\gamma_{i} \tau_{i}} \overline{D_{i}}-1\right) .
$$

Then, the trivial equilibrium of the impulsive system (1.9) is globally asymptotically stable.

\subsection{Periodic impulsions and existence of periodic solution}

In this section, we consider system (1.9) with periodic impulsions. Thus, we assume that

(H4) The function $t \rightarrow \prod_{0<t_{k}<t}\left(1+b_{k}\right)$ is periodic on the interval $[0,+\infty)$ with period $\omega>0$.

Remind that the function $t \rightarrow \prod_{0<t_{k}<t}\left(1+b_{k}\right)$ is equal to one for $t \leq 0$ and also for $t=p \omega$, for $p \in \mathbb{N}$.

In this part, we prove that there exists a unique positive $\omega$-periodic solution $x^{*}(t)$ of system (1.9). More precisely, we have the following result.

Theorem 9. Let the assumptions $(\boldsymbol{H 1})-(\boldsymbol{H} 4)$ and the inequality

$$
\delta<\beta_{0} \frac{\bar{A}^{n}}{\bar{A}^{n}}\left(2 \sum_{i=1}^{m} \alpha_{i} e^{-\gamma_{i} k_{i} \omega} \underline{D_{i}}-1\right)
$$

be satisfied, and $\tau_{i}=k_{i} \omega$, for $i=1, \ldots, m$, where $\left(k_{i}\right)_{i=1, \ldots, m}$ are some positive integers and $\underline{D_{i}}=\inf _{t>0}\left\{d_{i}(t)\right\}$. Then, there exists a unique $\omega$-periodic solution of (1.9).

Remark 10. The assumption $\tau_{i}=k_{i} \omega$ corresponds to a concept thus takes advantage of rhythmicity of the cell-cycle to see the effect of $\omega$-periodic impulsions on the system.

The proof of this result relies on some properties of the following ordinary differential system

$$
\left\{\begin{array}{l}
x^{\prime}(t)=-(\delta+\beta(x(t))) x(t)+\left(2 \sum_{i=1}^{m} \alpha_{i} e^{-\gamma_{i} k_{i} \omega}\right) \beta(x(t)) x(t), \\
x\left(t_{k}^{+}\right)=\left(1+b_{k}\right) x\left(t_{k}\right), \quad k=1,2, \ldots
\end{array}\right.
$$


or equivalently the following non-autonomous ordinary differential equation

$$
y^{\prime}(t)=-\delta y(t)+\beta_{0} \frac{a^{n}(t)}{a^{n}(t)+y^{n}(t)}\left(2 \sum_{i=1}^{m} \alpha_{i} e^{-\gamma_{i} k_{i} \omega} d_{i}(t)-1\right) y(t) .
$$

Remark that as the function $t \rightarrow \prod_{0<t_{k}<t}\left(1+b_{k}\right)$ is periodic on the interval $[0,+\infty)$ with period $\omega>0$, we have

$$
d_{i}(t)=\left\{\begin{array}{l}
\frac{a(t)}{\theta}, \quad \text { if } t<k_{i} \omega \\
1, \quad \text { if } t \geq k_{i} \omega
\end{array}\right.
$$

Now, we give sufficient conditions for the existence of $\omega$-periodic solution $x^{*}(t)$ of (3.8).

Lemma 11. Let the assumptions (H1)-(H4) be satisfied together with the inequality (3.7). Then, there exists a unique positive $\omega$-periodic solution $x^{*}(t)$ of system (3.8). Moreover, any nonnegative solution $t \rightarrow x(t)$ is attracted by the periodic solution. This means

$$
\lim _{t \rightarrow+\infty}\left(x(t)-x^{*}(t)\right)=0 .
$$

Proof. To prove the lemma, it suffices to prove that equation (3.9) has a unique positive and attractive $\omega$-periodic solution $y^{*}(t)$. First, we use the classical Brouwer's fixed point theorem to prove the existence of $x^{*}$. For that purpose, we consider the two following functions

$$
f_{1}(z)=-\delta+\beta_{0} \frac{\underline{A}^{n}}{\bar{A}^{n}+z^{n}}\left(2 \sum_{i=1}^{m} \alpha_{i} e^{-\gamma_{i} k_{i} \omega} \underline{D_{i}}-1\right)
$$

and

$$
f_{2}(z)=-\delta+\beta_{0} \frac{\bar{A}^{n}}{\underline{A}^{n}+z^{n}}\left(2 \sum_{i=1}^{m} \alpha_{i} e^{-\gamma_{i} k_{i} \omega} \overline{D_{i}}-1\right) .
$$

First, note that $\lim _{z \rightarrow \infty} f_{1}(z)=\lim _{z \rightarrow \infty} f_{2}(z)=-\delta<0$. Then, due to (3.7) we have $f_{2}(0)>f_{1}(0)>0$. Thus, we conclude that the equation $f_{1}(z)=0$ (resp. $f_{2}(z)=0$ ) has a unique positive root $z_{1}$ (resp. $z_{2}$ ), that is, $f_{1}\left(z_{1}\right)=0$ (resp. $f_{2}\left(z_{2}\right)=0$ ). In addition, we have $f_{1}(z)<f_{2}(z)$, for all $z>0$. Then $0<z_{1}<z_{2}$. It should be pointed out that the function $f_{1}$ is positive (resp. negative) on the interval $\left[0, z_{1}\right)$ (resp. on $\left(z_{1},+\infty\right)$ ) while the function $f_{2}$ is positive (resp. negative) on $\left[0, z_{2}\right)$ (resp. on $\left(z_{2},+\infty\right)$ ). For any $z_{0}>0$, we denote by $y\left(t, 0, z_{0}\right)$ the solution of (3.9) with initial value $z_{0}$. We claim that if $z_{0} \in\left[z_{1}, z_{2}\right]$ then the solution $y \equiv y\left(., 0, z_{0}\right)$ satisfies $y\left(t, 0, z_{0}\right) \in\left[z_{1}, z_{2}\right]$ for any $t>0$. Suppose that there exists $t_{1}>0$ such that $y\left(t_{1}\right)>z_{2}$. Let $t^{*}$ be defined by $t^{*}=\inf \left\{t>0: y(t)>z_{2}\right\}$. Then there exists a $\bar{t} \geq t^{*}, \bar{t} \neq t_{k}$ such that $y(\bar{t})>z_{2}$ and 
$y^{\prime}(\bar{t}) \geq 0$. Since $y(\bar{t})>z_{2}$, we derive from (3.9) that

$$
\begin{aligned}
y^{\prime}(\bar{t}) & =-\delta y(\bar{t})+\beta_{0} \frac{a^{n}(\bar{t})}{a^{n}(\bar{t})+y^{n}(\bar{t})}\left(2 \sum_{i=1}^{m} \alpha_{i} e^{-\gamma_{i} k_{i} \omega} d_{i}(t)-1\right) y(\bar{t}), \\
& \leq y(\bar{t})\left[-\delta+\beta_{0} \frac{\bar{A}^{n}}{\underline{A}^{n}+y^{n}(\bar{t})}\left(2 \sum_{i=1}^{m} \alpha_{i} e^{-\gamma_{i} k_{i} \omega} \overline{D_{i}}-1\right)\right], \\
& =y(\bar{t}) f_{2}(y(\bar{t})), \\
& <y(\bar{t}) f_{2}\left(z_{2}\right), \\
& =0 .
\end{aligned}
$$

This contradicts the fact that $y^{\prime}(\bar{t}) \geq 0$ and proves the claim result. Using a similar argument, we can show that $y(t) \geq z_{1}$ for all $t \geq 0$. Hence, in particular, $y(\omega)=y\left(\omega, 0, z_{0}\right) \in\left[z_{1}, z_{2}\right]$ for any $z_{0} \in\left[z_{1}, z_{2}\right]$.

We define a mapping $G:\left[z_{1}, z_{2}\right] \rightarrow\left[z_{1}, z_{2}\right]$ as follows: for each $z_{0} \in\left[z_{1}, z_{2}\right], G\left(z_{0}\right)=$ $y\left(\omega, 0, z_{0}\right)$. Since the solution $y(t)=y\left(t, 0, z_{0}\right)$ depends continuously on the initial value $z_{0}$, it follows that $G$ is a continuous function and maps the interval $\left[z_{1}, z_{2}\right]$ into itself. By the Brouwer's fixed point theorem, $G$ has a fixed point $z_{0}^{*}$. Then, $y^{*}\left(\omega, 0, z_{0}^{*}\right)=G\left(z_{0}^{*}\right)=z_{0}^{*}$. This means that the positive function $y^{*}$ is an $\omega$-periodic solution of (3.9). In view of the periodicity of $t \rightarrow \prod_{0<t_{k}<t}(1+$ $b_{k}$ ) on $[0,+\infty)$, it follows that the positive function $x^{*}$ defined by $x^{*}(t)=\prod_{0<t_{k}<t}\left(1+b_{k}\right) y^{*}(t)$ is an $\omega$-periodic solution of (3.8). The uniqueness of this solution will be a consequence of its attractiveness. We now consider the attractiveness of the periodic solution constructed above. Let $x^{*}$ be a $\omega$-periodic positive solution of (3.8). Then, $t \rightarrow y^{*}(t)=\prod_{0<t_{k}<t}\left(1+b_{k}\right)^{-1} x^{*}(t)$ is an $\omega$ periodic positive solution of (3.9). Let $t \rightarrow x(t)$ be any other positive solution of (3.8). We consider the corresponding solution of (3.9), that is, $t \rightarrow y(t)=\prod_{0<t_{k}<t}\left(1+b_{k}\right)^{-1} x(t)$. As the functions $y^{*}$ and $y$ are continuous and by the uniqueness property of the solutions of (3.9), we conclude that the map $t \rightarrow y(t)$ never cross the map $t \rightarrow y^{*}(t)$. Then, we can assume that $y(t)>y^{*}(t)$ for $t>0$. Note that the case where $y(t)<y^{*}(t)$ for $t>0$ can be handled with similar arguments.

Set

$$
y(t)=y^{*}(t) e^{v(t)}, \quad t>0
$$

Then, $v(t)>0$ for $t>0$ and we have

$$
\begin{aligned}
v^{\prime}(t) & =\left(\ln \frac{y(t)}{y^{*}(t)}\right)^{\prime}=\frac{y^{\prime}(t)}{y(t)}-\frac{y^{*^{\prime}}(t)}{y^{*}(t)} \\
& =\beta_{0}\left(2 \sum_{i=1}^{m} \alpha_{i} e^{-\gamma_{i} k_{i} \omega} d_{i}(t)-1\right) \frac{a^{n}(t) y^{* n}(t)\left[1-e^{n v(t)}\right]}{\left[a^{n}(t)+y^{* n}(t) e^{n v(t)}\right]\left[a^{n}(t)+y^{* n}(t)\right]} .
\end{aligned}
$$

Thanks to (3.7), we know that $2 \sum_{i=1}^{n} e^{-\gamma_{i} k_{i} \omega} d_{i}(t)-1>0, t>0$. Note that $1-e^{n v(t)}<0$ for $t>0$. It follows that $v^{\prime}(t)<0$. Thus $t \rightarrow v(t)$ is decreasing and therefore $\lim _{t \rightarrow \infty} v(t)=L \in[0,+\infty)$. 
Now we shall prove that $L=0$. For that purpose, let us suppose that $L>0$. Then there exist $0<\alpha<L$ and $T>0$ such that for $t \geq T>0, L-\alpha<v(t)<L+\alpha$. On the other hand, as $1-e^{n v(t)}<0$ and using (3.12) for $t \geq T$, we get

$$
v^{\prime}(t) \leq \beta_{0}\left(2 \sum_{i=1}^{m} \alpha_{i} e^{-\gamma_{i} k_{i} \omega} \underline{D_{i}}-1\right) \frac{\underline{A}^{n}\left(\underline{Y}^{*}\right)^{n}}{\left(\bar{A}^{n}+\left(\bar{Y}^{*}\right)^{n} e^{n(L+\alpha)}\right)\left(\bar{A}^{n}+\left(\bar{Y}^{*}\right)^{n}\right)}\left(1-e^{n(L-\alpha)}\right),
$$

where

$$
\bar{Y}^{*}=\sup _{t \geq 0}\left\{y^{*}(t)\right\}, \quad \underline{Y}^{*}=\inf _{t \geq 0}\left\{y^{*}(t)\right\} .
$$

By integrating (3.13) from $T$ to $t>T$ we obtain

$$
v(t)-v(T) \leq H\left(1-e^{n(L-\alpha)}\right)(t-T),
$$

where $H$ is a positive constant given by

$$
H=\beta_{0}\left(2 \sum_{i=1}^{m} \alpha_{i} e^{-\gamma_{i} k_{i} \omega} \underline{D_{i}}-1\right) \frac{\underline{A}^{n}\left(\underline{Y}^{*}\right)^{n}}{\left(\bar{A}^{n}+\left(\bar{Y}^{*}\right)^{n} e^{n(L+\alpha)}\right)\left(\bar{A}^{n}+\left(\bar{Y}^{*}\right)^{n}\right)} .
$$

When $t \rightarrow+\infty$, we immediately lead to a contradiction. Thus, $L=0$ and therefore $t \rightarrow v(t)$ tends to zero as $t \rightarrow 0$. Consequently, we obtain

$$
\lim _{t \rightarrow \infty}\left(x(t)-x^{*}(t)\right)=\lim _{t \rightarrow \infty}\left[y^{*}(t) \prod_{0<t_{k}<t}\left(1+b_{k}\right)\left(e^{v(t)}-1\right)\right]=0 .
$$

Finally, the attractiveness of this periodic solution implies the uniqueness of non-negative and $\omega$-periodic solution of equation (3.9). The proof is complete.

We now come on the proof of Theorem 9.

Proof of Theorem 9. In view of Lemma 11, equation (3.9) has a unique $\omega$-periodic solution $y^{*}(t)$. However, $y^{*}(t)$ is also an $\omega$-periodic solution of (3.2). Thus, by Lemma 6 the map $t \rightarrow x^{*}(t)=$ $\prod_{0<t_{k}<t}\left(1+b_{k}\right) y^{*}(t)$ is an $\omega$-periodic solution of (1.9). On the other hand, if $x^{*}(t)$ is an $\omega$-periodic and positive solution of (1.9), it is also a positive and $\omega$-periodic solution of (3.9). Finally, by Lemma 11, the $\omega$-periodic solution of (1.9) is unique.

\section{Numerical simulations}

This section is devoted to numerical simulations for equation (2.1). We only consider one delay, that is $m=1$. This means that there is only one compartment of proliferating phase. The corresponding delay will be simply denoted by $\tau$ instead of $\tau_{1}$. We consider some fixed parameters

$$
\beta_{0}=1.77 \mathrm{day}^{-1}, \quad \gamma=\gamma_{1}=0.1 \mathrm{day}^{-1}, \quad \theta=1, \quad \alpha_{1}=1, \quad n=3 .
$$


Next we investigate different situations depending on the behavior of the equation without impulsion. Then we add some impulsions to see their action on the system.

We first consider the case where the population of cells goes to extinction without impulsion and we add only one impulsion with different intensities.
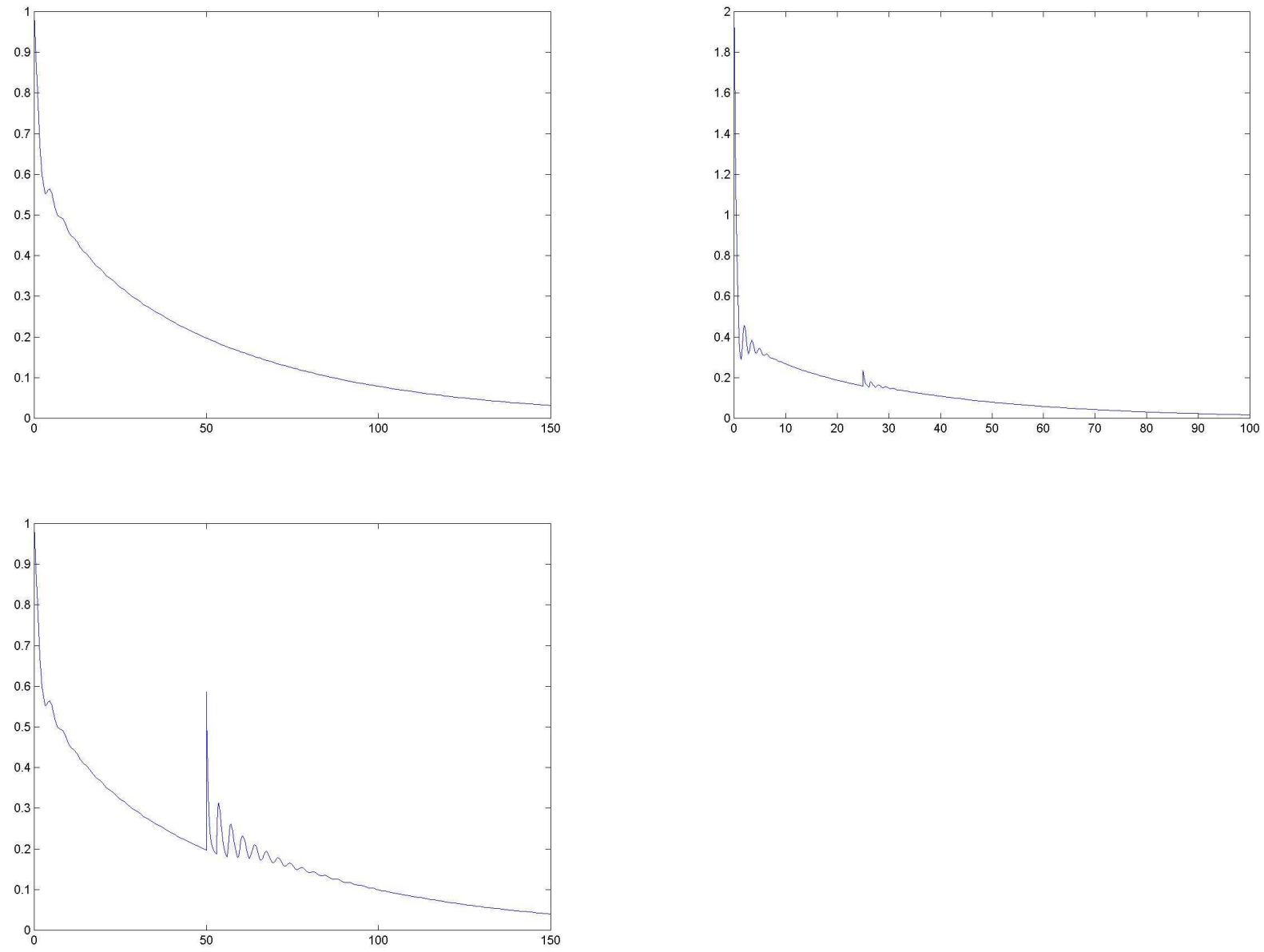

Figure 1: Evolution of the population of cells $x(t)$ in function of time with $\tau=3$ days and $\delta=0.3$ day $^{-1}$. The population goes to zero without impulsion (up left). Different intensities of one impulsion, $b_{1}=0.8$ (up right) and $b_{1}=2$ (bottom), are given.

Next, we consider the case where the population of cells without impulsion stabilizes to a non trivial steady state. 

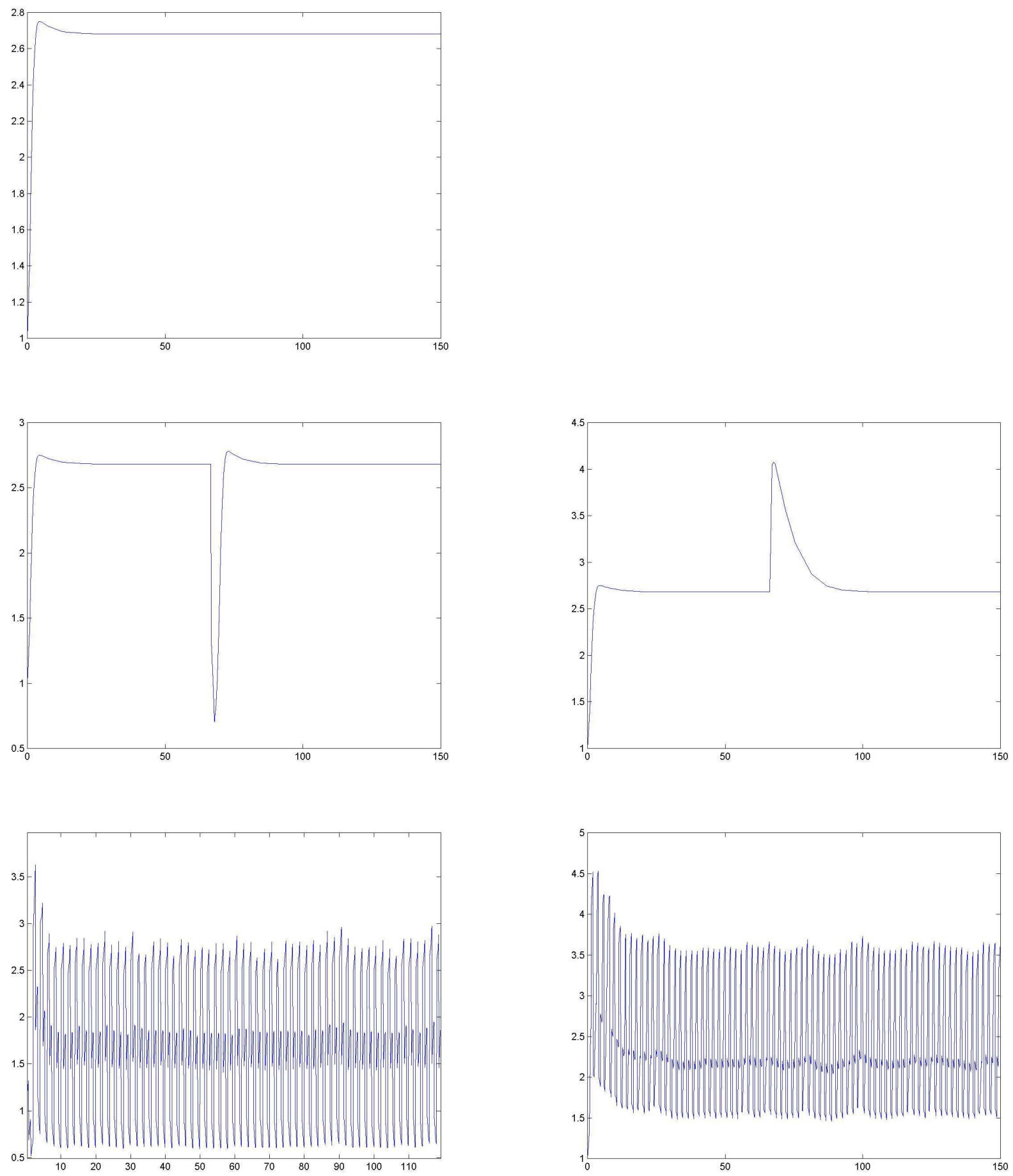

Figure 2: Evolution of the population of cells $x(t)$ in function of time with $\tau=1.2$ day, $\gamma=0.2$ day $^{-1}$ and $\delta=0.05$ day $^{-1}$. The population goes to a positive equilibrium without impulsion (up left). Different intensities of one impulsion, $b_{1}=-0.5$ (middle left) and $b_{1}=0.5$ (middle right) are 
considered. $\omega$-Periodic impulsions with $\omega=2$ days and two impulsions per period with identical intensities, $b_{1}=b_{2}=-0.5$ (bottom left), $b_{1}=b_{2}=0.5$ (bottom right) and $b_{1}=5$ (bottom) are given.
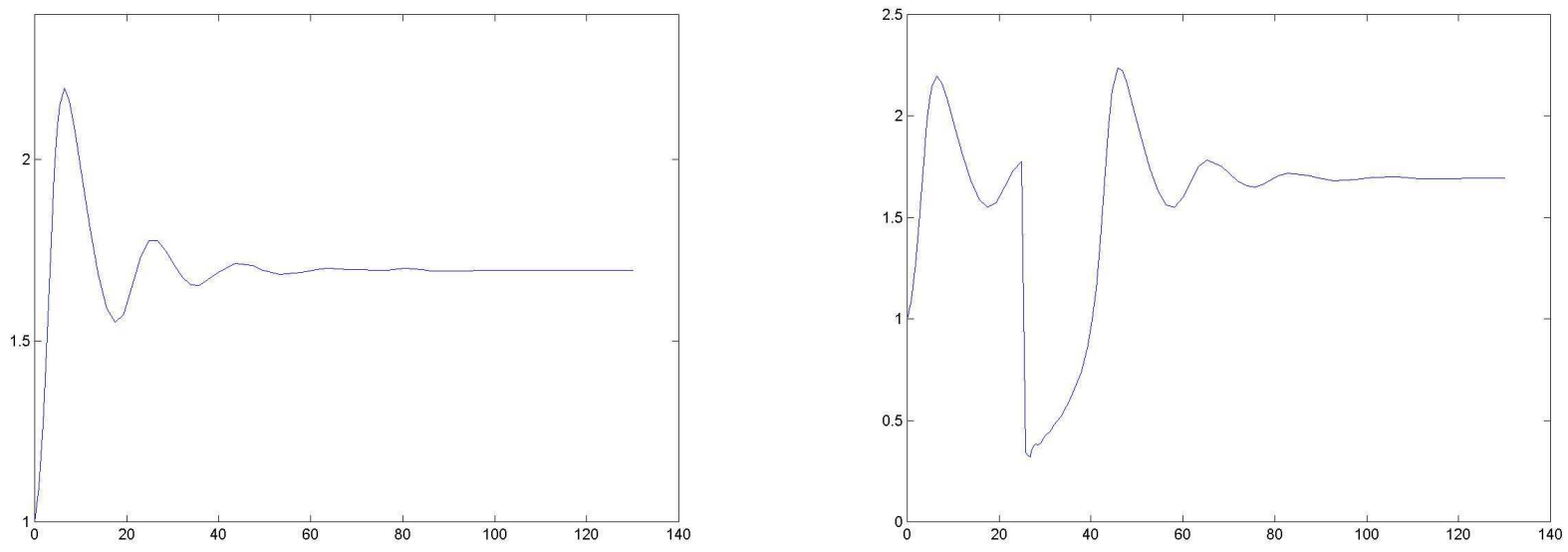

Figure 3: Evolution of the population of cells $x(t)$ in function of time with $\tau=1.8$ day, $\gamma=0.3$ day $^{-1}$ and $\delta=0.05$ day $^{-1}$. The population goes to a positive equilibrium: without impulsion (left) and with one impulsion with intensity $b_{1}=-0.8$ (right).

The action of only one impulsion does not destabilized the dynamic of the system with impulses. As shown in Figures 1, 2 and 3, we observe that after one impulse, the system comes back to its original behavior (with overshot). The application of an infinite number of impulsions (in particular periodic impulsions) can generate a more complex dynamics as the ones presented in Figures 2 and 4. The dynamics seems to exhibit a non-periodic dynamics when the parameters are far from the assumptions of Theorem 9. However, the application of negative periodic impulses $\left(b_{k}<0\right)$ allows to keep the density of stem cells quite low during a certain time. After that, the system recovers its initial behavior due to the attractiveness of stationary or periodic solutions. 

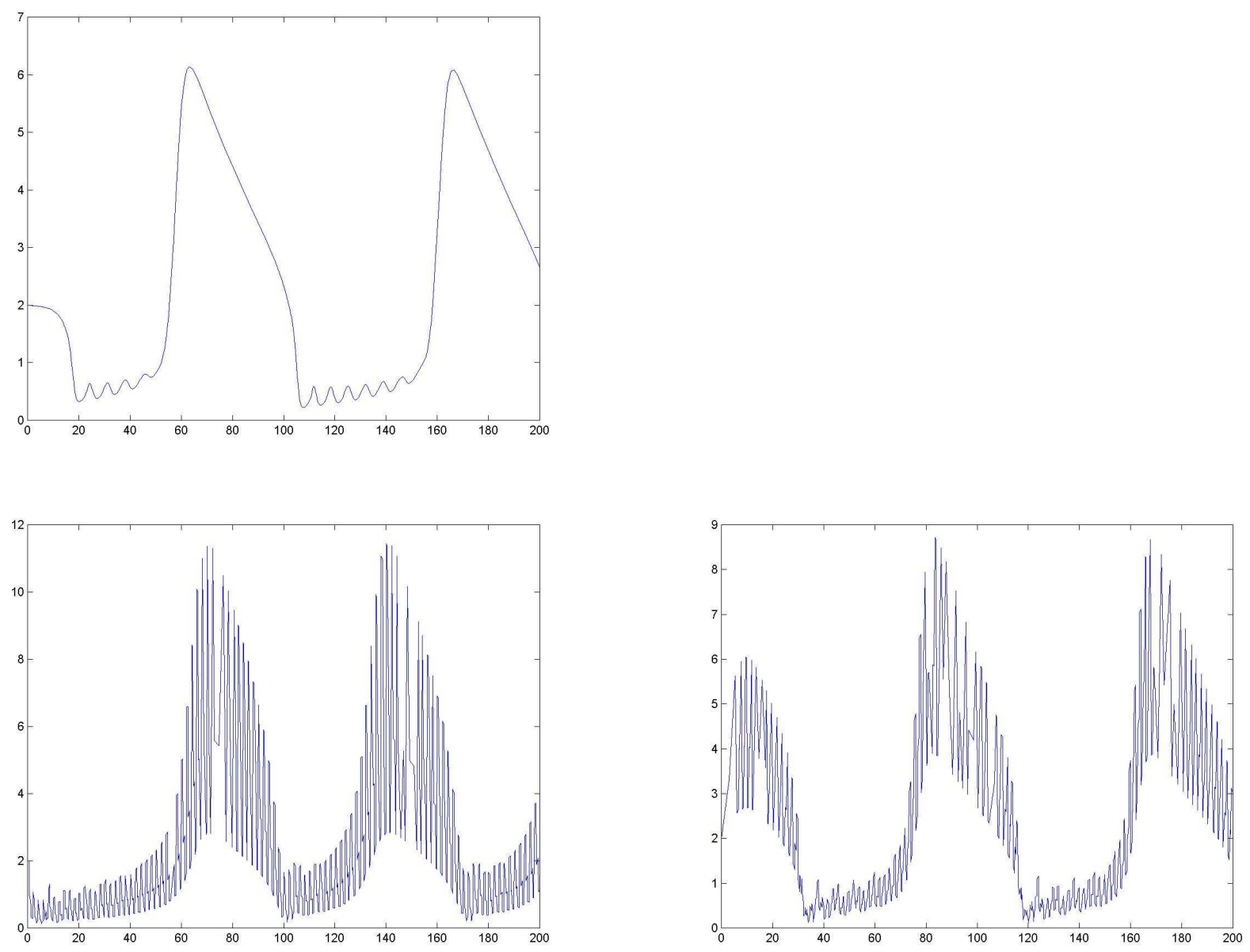

Figure 4: Evolution of $x(t)$ in function of time with $\tau=6$ days and $\delta=0.02$ day $^{-1}$ : without impulsion (up left), with $\omega$-periodic ( $\omega=2$ days) impulsions and with two identical impulsions per period $b_{1}=b_{2}=-0.5$ (bottom left), $b_{1}=b_{2}=0.5$ (bottom right).

\section{Conclusion}

In this paper, we proposed a mathematical model of hematopoietic stem cell dynamics with impulse perturbations. The resulting age-structured model was reduced to a delay differential equation with impulses. The delay equation without impulses was studied. Existence and stability of steady states were investigated. We proposed a Lyapunov functional to prove the global asymptotic stability of the trivial steady state. This equilibrium describes cell population extinction. We showed that the instability of this equilibrium occurs when a positive steady state appears (the dynamical system undergoes a transcritical bifurcation). Then, we obtained a sufficient condition for the local asymptotic stability of the positive steady state. The second part of this work has 
been devoted to the influence of impulsive perturbations on the model. We transformed the delay differential equation with impulses into a non-autonomous delay differential equation without impulsion. We proved positivity and boundedness properties of the solutions and we obtained a sufficient condition for the global stability of the zero solution using Lyapunov function. In the third part, we gave some results concerning the influence of periodic impulses on the delay differential equation. Then we focused on the existence and uniqueness of the periodic solution. Finally, numerical simulations have been fulfilled in order to understand the dynamics with conditions far from our mathematical assumptions. As a conclusion, we obtained that the application of impulses does not allow the total destabilization of the dynamic of the model. This property seems to be a consequence of the stability of the steady states in the case of non impulsive equation: the model without impulsion exhibits a monostable dynamics (with only one attractor) and does not allow, in the case of impulsive perturbations, the total destabilization of the dynamics of the system. However, the infinite number of impulsions can generate some non-periodic oscillations and complex dynamics with high amplitudes.

\section{References}

[1] M. Adimy, F. Crauste. Global stability of a partial differential equation with distributed delay due to celluar replication. Nonlinear Analysis, 54 (2003), No. 8, 1469-1491.

[2] M. Adimy, F. Crauste. Existence, positivity and stability for a nonlinear model of celluar proliferation. Nonlinear Analysis: Real World Applications, 6 (2005), No. 2, 337-366.

[3] M. Adimy, F. Crauste, L. Pujo-Menjouet. On the stability of a maturity structured model of cellular proliferation. Discret. Cont. Dyn. Sys. Ser. A, 12 (2005), No. 3, 501-522.

[4] M. Adimy, F. Crauste, S. Ruan. A mathematical study of the hematopoiesis process with applications to chronic myelogenous leukemia. SIAM J. Appl. Math., 65 (2005), No. 4, 13281352 .

[5] M. Adimy, Pujo-Menjouet. Asymptotic behaviour of a singular transport equation modeling cell division. Discret. Cont. Dyn. Sys. B, 3 (2003), No. 3, 439-456.

[6] S. Bernard, J. Belair, M.C. Mackey. Oscillations in cyclical neutropenia: New evidence based on mathematical modeling. J. Theor. Biol., 223 (2003), No. 3, 283-298.

[7] S. Bernard, J. Belair, M.C. Mackey. Bifurcations in a white-blood-cell production model. C. R. Biologies, 327 (2004), No. 3, 201-210.

[8] F.J. Burns, I.F. Tannock. On the existence of a $G_{0}$ phase in the cell cycle. Cell. Tissue Kinet., 19 (1970), No. 4, 321-334.

[9] C. Colijn, M.C. Mackey. A mathematical model of hematopoiesis, I. Periodic chronic myelogenous leukemia. J. Theor. Biol., 237 (2005), No. 2, 117-132. 
[10] C. Colijn, M.C. Mackey. A mathematical model of hematopoiesis, II. Cyclical neutropenia. J. Theor. Biol., 237 (2005), No. 2, 133-146.

[11] J.J. Ferrell. Tripping the switch fantastic: How protein kinase cascade convert graded into switch-like outputs. TIBS, 21 (1996), No. 12, 460-466.

[12] K. Gopalsamy, B.G. Zhang. On delay differential equation with impulses. J. Math. Anal. Appl., 139 (1989), No. 11, 110-122.

[13] I. Gyori, G. Ladas. Oscillation theory of delay differential equations with applications. Clarendon, Oxford, 1991.

[14] J. Hale, S.M. Verduyn Lunel. Introduction to functional differential equations. Applied Mathematical Sciences 99. Springer-Verlag, New York, 1993.

[15] C. Haurie, D.C. Dale, M.C. Mackey. Cyclical neutropenia and other periodic hematological diseases: A review of mechanisms and mathematical models. Blood, 92 (1998), No. 8, 26292640 .

[16] Y. Kuang. Delay differential equations with application in population dynamics. Academic Press. Boston, MA, 1993.

[17] V. Lakshmikantham, D.D. Bainov, P.S. Simeonov. Theory of impulsive differential equations. World Scientific. Singapore, 1989.

[18] M. Loeffler, H.E. Wichmann. A comprehensive mathematical model of stem cell proliferation which reproduces most of the published experimental results. Cell Tissue Kinet., 13 (1980), No. 5, 543-561.

[19] M.C. Mackey. Unified hypothesis of the origin of aplastic anaemia and periodic hematopoiesis. Blood, 51 (1978), No. 5, 941-956.

[20] M.C. Mackey. Dynamic hematological disorders of stem cell origin. In Biophysical and Biochemical Information Transfer in Recognition, J.G. Vassileva-Popova and E.V. Jensen, eds., Plenum Publishing, New York, 1979, 373-409.

[21] M.C. Mackey. Mathematical models of hematopoietic cell replication and control. in The Art of Mathematical Modelling: Case Studies in Ecology, Physiology and Biofluids, PrenticeHall, Upper Saddle River, NJ, 1997, 149-178.

[22] M.C. Mackey, L. Pujo-Menjouet, J. Wu. Period oscillations of blood cell populations in periodic myelogenous leukemia. SIAM J. Math. Anal., 38 (2006), No. 1, 166-187.

[23] M.C. Mackey, A. Rey. Propagation of population pulses and fronts in a cell replication problem: non-locality and dependence on the initial function. Physica D, 86 (1995), No. 3, 373395. 
[24] M.C. Mackey, R. Rudnicki. Global stability in a delayed partial differential equation describing cellular replication. J. Math. Bio., 33 (1994), No. 1, 89-109.

[25] L. Pujo-Menjouet, M.C. Mackey. Contribution to the study of periodic chronic myelogenous leukemia. C. R. Biologies, 327 (2004), No. 3, 235-244.

[26] L. Pujo-Menjouet, S. Bernard, M.C. Mackey. Long Period Oscillations in a $G_{0}$ Model of Hematopoietic Stem Cells. SIAM J. Appl. Dynam. Systems, 4 (2005), No. 2, 312-332.

[27] S.I. Rubino, J.L. Lebowitz. A mathematical model of neutrophil production and control in normal man. J. Math. Bio., 1 (1975), No. 3, 187-225.

[28] L. Sachs. The molecular control of hemopoiesis and leukemia. C. R. Acad. Sci. Paris, 316 (1993), No. 9, 882-891.

[29] S.H. Saker, J.O. Alzabut. Existence of periodic solutions, global attractivity and oscillation of impulsive delay population model. Nonlinear Analysis: real world applications, 8 (2007), No. 4, 1029-1039.

[30] G.F. Webb. Theory of Nonlinear Age-dependent Population Dynamics. Monogr. Textbooks Pure Appl. Math., 89, Dekker, New York, 1985.

[31] J. Yan, A. Zhao. Oscillation and stability of linear impulsive delay differential equations. J. Math. Anal. Appl., 227 (1998), No. 1, 187-194.

[32] J. Yan, A. Zhao, J.J. Nieto. Existence and global attractivity of positive periodic solution of periodic single-species impulsive Lotka-Volterra Systems. Mathematical and Computer Modelling, 40 (2004), No 5-6, 509-518. 